\section{Stanley Whittingham (Binghamton University, USA)}

\section{Abstract}

During the past two decades, the demand for the storage of electrical energy has mushroomed both for portable applications and for static applications. As storage and power demands have increased predominantly in the form of batteries, the system has evolved. However, the present electrochemical systems are too costly to penetrate major new markets, still higher performance is required, and environmentally acceptable materials are preferred. These limitations can be overcome only by major advances in new materials whose constituent elements must be available in large quantities in nature; nanomaterials appear to have a key role to play. New cathode materials with higher storage capacity are needed, as well as safer and lower cost anodes and stable electrolyte systems. Flywheels and pumped hydropower also have niche roles to play.

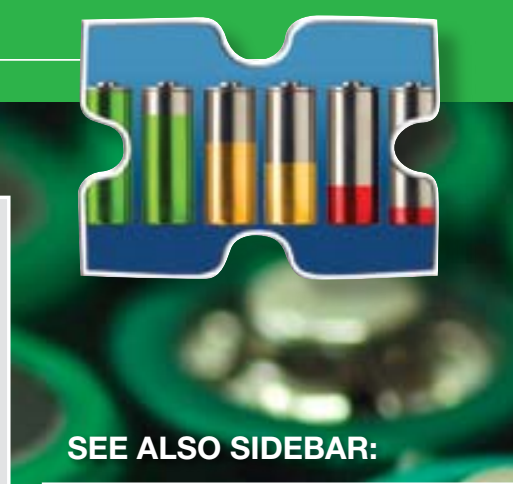

Flywheels

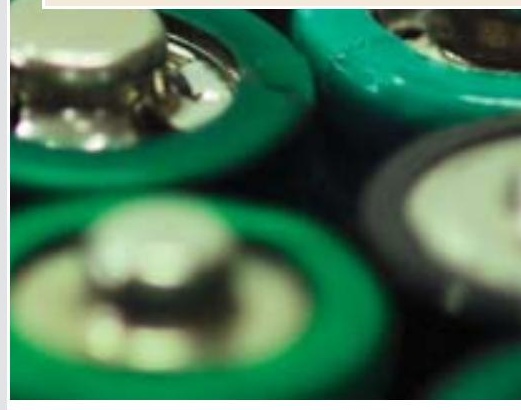

\section{Introduction to Energy Storage}

Why store energy, and in particular, why store electrical energy? There is a great need for electrical energy storage, not only for mobile electronic devices, such as cell phones, computers, and iPods, but also for transportation and load-leveling and for the effective commercialization of renewable resources such as solar and wind power. The storage sizes needed range from milliwatts for smart-card devices to multiple-megawatts for large load-leveling stations; unlike for electronics, there is no simple applicable Moore's law (i.e., no general trend of exponentially increasing storage capacity). Much attention is being given to hybrid electric vehicles (HEVs), in which batteries and/or capacitors are used to capture the energy evolved in braking. A related application is the capture of the energy normally wasted when a dock crane is lowering a crate; capturing this energy through capacitors saves around $40 \%$ of total energy utilization. Similarly, subway trains, with many stops and starts, can capture the braking energy entering the station for use in accelerating out of the station. The next generation of electric vehicles might be plug-in hybrids, in which larger batteries are used and the vehicle can be recharged by plugging into the electrical power line. An effective unlimited range can be attained by using a small internal engine as a battery charger; the waste heat from the internal combustion engine can provide the heating and defogging energy. The all-electric vehicle might find a few niche markets; these include city buses and postal delivery and utility repair vehicles with much stopping and starting and limited daily ranges, high-cost hot-rod sports cars, and small commuter cars. In all of these transportation applications, low cost and long life are essential for commercial success. Neither can be attained with the present chemical storage battery and capacitor charge storage systems.

An application demanding even lower costs and higher reliability is in load-leveling and central back-up. A typical daily electrical usage cycle is shown in Figure 1. There is considerable hourly variation, and the load could be leveled by, for example, storing energy during the early morning low-usage hours and using that energy during the peak hours of 4-6 pm. This would not only reduce the total generating capacity needed,

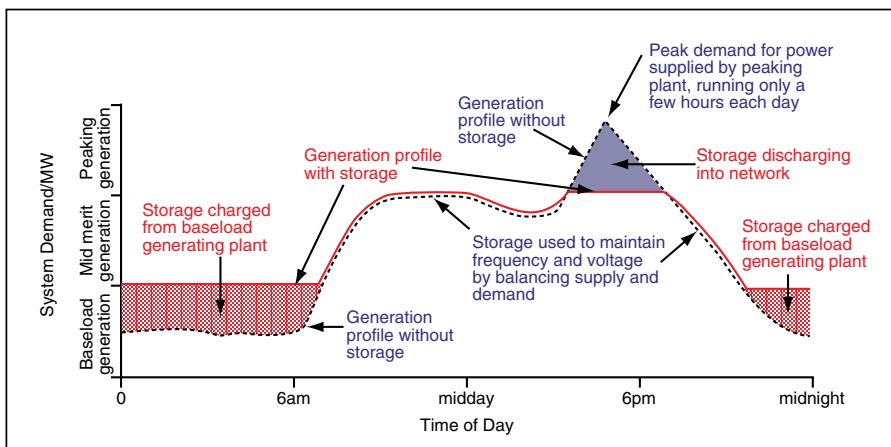

Figure 1. Typical electrical power profile, showing the large variations during a 24-h period. In a load-leveling scenario, an electrical energy storage device would be charged during periods of low power demand and would discharge during periods of high power demand, thus filling in the valleys and cutting off the peaks. A utility would thus need less overall power generation capability and could delay the installation of extra capacity for a period.

but make the remaining generation capacity more efficient. Here, the system is static and can range from a few kilowatthours for a home solar energy system to many megawatt-hours for a utility load-leveling application. The former are satisfied today by lead-acid batteries, and the latter by a range of systems ranging from $\mathrm{Ni}-\mathrm{Cd}$ through redox systems to advanced sodium-sulfur systems. The last demonstrates how long a new technology takes to come to market. The sodium-sulfur battery, conceived by the Ford Motor Company, uses the solid electrolyte sodium- $\beta$-alumina, whose exceptional ionic conductivity properties were reported in 1967. After almost 40 years of development mostly in Europe, NGK Insulators commercialized the battery for load leveling in Japan; a 1 MW NGK battery is used on Long Island, New York, to provide power during peak periods for compressors for 220 natural gas buses. Vanadium redox batteries are being used for supplementing peak power needs in 
Castle Valley, Utah, as well as for load-leveling wind farms and other applications throughout the world; a $40 \mathrm{MW} \mathrm{Ni-Cd} \mathrm{bat-}$ tery system is used in Fairbanks, Alaska, for guaranteeing continuous power availability. To enable renewable energy, which is often intermittent in nature, localized energy storage is desirable. To place the economic desire for uninterruptable power in context, some $\$ 80$ billion is lost by industry in the United States ${ }^{1}$ each year because of mainly short power interruptions. To ameliorate this problem, high-tech, high-cost industry such as chip fabrication plants have large power storage backups and employ frequency smoothing, using, for example, lead-acid batteries.

Aside from batteries and capacitors, there are a number of other options, such as water pumping, flywheels, and superconducting magnetic energy storage (SMES) systems. In the former, water is pumped uphill into "reservoirs" when excess generating capacity is available and then allowed to flow downhill through the pump turbines during high peak demand. One such example is found in the Snowdonia mountains of Wales at Dinorwig. ${ }^{2}$ This system has $288 \mathrm{MW}$ of power output. There are, however, limited locations where water-pumping can be installed. A variation on water-pumping is to use excess electricity to compress air and allow the compressed air to drive turbines during peak hours; such a facility is in the planning stage in Iowa in conjunction with wind and off-peak power. Here again, the technology is limited by the availability of suitable caverns. Ultracapacitors are finding application for wind smoothing and backup power, for example, at the Palmdale, California water treatment facility. Flywheels (see the accompanying sidebar) and capacitors have found application for grid frequency regulation on critical applications, as utilities commonly vary the frequency to smooth the power output. The proposed hydrogen economy uses hydrogen as an energy source; this hydrogen can be formed from fossil fuels or grains or by electrolysis from water. SMES, an elegant storage means, is no longer under serious consideration because of cost and technical limitations.

To place in perspective the energy storage capabilities of these options, Table I provides a comparison, from which it can be seen that the systems being considered have much inferior

\section{Table I: Energy Output of Electrochemical Devices and Petroleum.}

Energy Source

\begin{tabular}{l|l|}
\hline Supercapacitors & 0.01 \\
\hline Lithium battery & 0.8 \\
\hline Hydrogen fuel cell & 1.1 \\
\hline Gasoline & $6.0^{\mathrm{a}}$ \\
\hline
\end{tabular}

assuming $30 \%$ combustion efficiency

energy storage capabilities compared to that of gasoline. A pure hydrogen fuel cell has a theoretical capacity that is comparable to that of a lithium battery, because hydrogen containers hold only $5 \mathrm{wt} \%$ hydrogen using the best storage media and high pressure, and because fuel cells put out just $0.8 \mathrm{~V}$.

The energy obtained from any storage device depends strongly not only on the device but also on the power output, as shown in Figure 2. Batteries can provide higher energies than capacitors, whereas capacitors are high-power devices with limited energy storage capability. In addition, the quality of the energy from capacitors is generally poor; that is, for capacitors the voltage delivered varies strongly with the state of discharge, whereas batteries tend to have a fairly constant output voltage. Fuel cells, operating on liquid fuels such as methanol, can have high energy storage, but their power output is limited. Moreover,

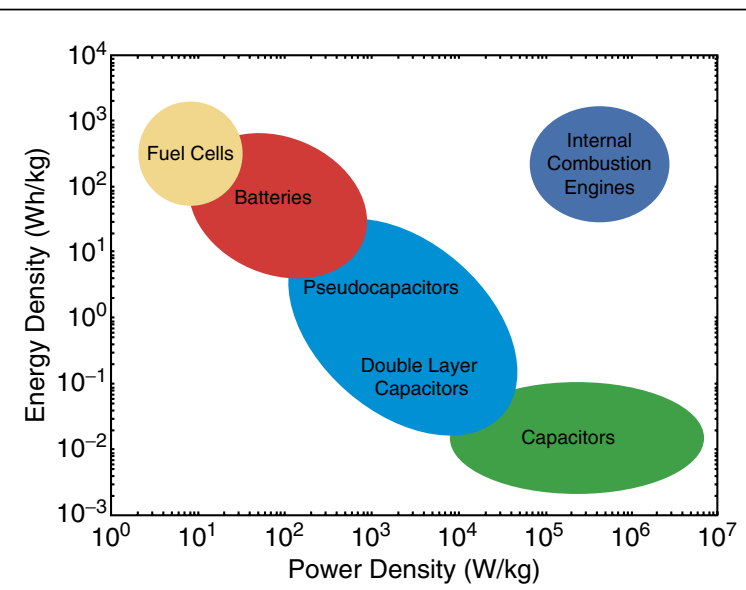

Figure 2. Comparison of the power density and energy density for batteries, capacitors, and fuel cells. (Energy is the capacity to do work; power is the rate at which work is done.)

their efficiency is optimum only at constant output, and their poor response time demands that they be coupled to a storage medium such as batteries.

Batteries, fuel cells, and capacitors have many features in common, all being based on electrochemistry. Pseudocapacitors can be described as high-power secondary batteries, and fuel cells can be described as primary batteries that are recharged/ refueled externally. Several reviews cover this area-batteries and fuel cells in References 3-6, energy storage in Reference 7, and electrochemical capacitors in Reference 8. An excellent introduction to batteries, fuel cells, and supercapacitors by Winter and Brodd can be found in Reference 3. Fuel cells are discussed elsewhere in this issue (see the article by Crabtree and Dresselhaus). Materials have always played a critical role in energy production, conversion, and storage, ${ }^{9}$ and they will continue to do so. This article discusses the materials challenges facing electrical energy storage, and a more extended discussion with extensive background material can be found in a 2007 U.S. Department of Energy (DOE) workshop on Electrical Energy Storage.?

\section{Chemical Energy Storage: Batteries}

Batteries are by far the most common form of storing electrical energy, and they range in size from the button cells used in watches to megawatt load-leveling applications. They are efficient storage devices, with output energy typically exceeding $90 \%$ of input energy, except at the highest power densities. Rechargable, also known as secondary, batteries have evolved over the years from lead-acid through nickel-cadmium and nickel-metal hydride (NiMH) to lithium-ion. NiMH batteries were the initial workhorse for electronic devices such as computers and cell phones, but they have almost been completely displaced from that market by lithium-ion because of the latter's higher energy storage capability. Today, NiMH technology is the principal battery used in hybrid electric vehicles, but it is likely to be displaced by the higher energy and now lower cost lithium batteries if the latter's safety and lifetime can be improved. The accompanying sidebar on batteries describes the different components of a modern-day rechargeable battery, all of which have materials limitations. Of the advanced batteries, lithium-ion, so called because the lithium ions shuttle back and forth between two intercalation electrodes, is the dominant power source for most rechargeable electronic devices. Initially developed in the early 1970s, the lithium-ion 
cell contains no lithium metal because of the difficulty of plating-out dendrite-free lithium on recharge; the formation of dendrites results in cell short-circuiting. Initially, lithium was alloyed with metals such as aluminum, which forms the 1:1 alloy LiAl with a very large energy storage capability (780 amp-hours per kilogram, $\mathrm{Ah} / \mathrm{kg}$ ). However, the volume of the aluminum increases by $200 \%$, which results in electrode crumbling, loss of electrical contact between particles, and rapid capacity loss. Carbon, however, can react with lithium very readily at room temperature to form the intercalation compound $\mathrm{LiC}_{6}$ and has been used in essentially all lithium batteries since $1990 ;{ }^{10}$ most recently, purified natural graphites have replaced the expensive synthetic carbons.

Although carbon has been the workhorse of lithium-ion batteries, its potential is only $300 \mathrm{mV}$ from that of lithium, which leads to possible problems on high-rate charging, such as in regenerative braking in HEVs, if the lithium intercalation rate into the carbon is too low. In such circumstances, the lithium ions can plate out as metallic lithium, which is commonly in the form of dendrites that can penetrate the separator, short the battery, and lead to thermal runaway and eventually a fire.

\section{What Is a Battery?}

A battery (see below) contains one or more electrochemical cells; these can be connected in series or parallel to provide the desired voltage and power.

The anode is the electropositive electrode from which electrons are generated to do external work. In a lithium cell, the anode contains lithium, commonly held within graphite in the well-known lithium-ion batteries.

The cathode is the electronegative electrode to which positive ions migrate inside the cell and to which electrons migrate through the external electrical circuit on discharge; during charging, the ions and electrons flow in the opposite directions.

The electrolyte allows the flow of the positive ions, for example, lithium ions from one electrode to another. It allows only the flow of ions and not the flow of electrons. The electrolyte is commonly a liquid solution containing a salt dissolved in a solvent. The electrolyte must be stable in the presence of both electrodes.

The current collectors allow the transport of electrons to and from the electrodes. They are usually metals and must not react with the electrode materials. Typically, copper is used for the anode and aluminum for the cathode (the lighter weight aluminum reacts with lithium and, therefore, cannot be used for lithiumbased anodes).

The cell voltage is determined by the energy of the chemical reaction occurring in the cell.

The anode and cathode are, in practice, complex composites, containing, in addition to the active material, polymeric binders to hold the powder structure together and conductive diluents such as carbon black to give the whole structure electronic conductivity so that electrons can be transported to the active material. Moreover, these components are combined in such a way as to leave sufficient porosity to allow the liquid electrolyte to penetrate the powder structure and the ions to reach the reacting sites.

(Adapted from Reference 7.)

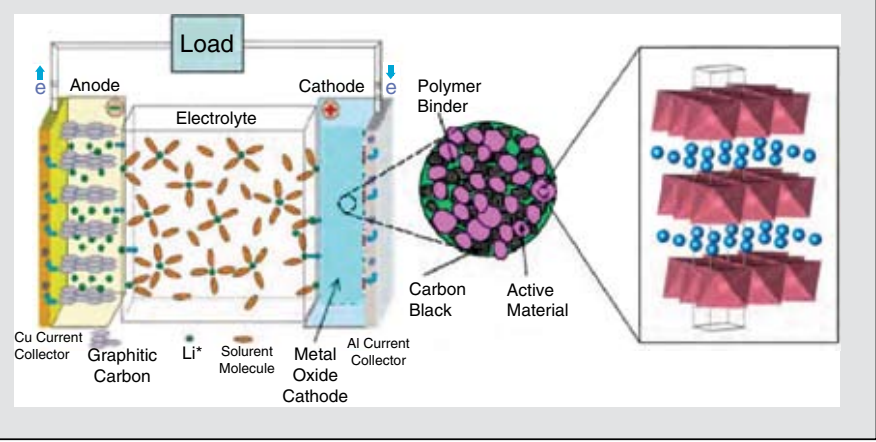

These carbon-lithium anodes also have low gravimetric and volumetric energy densities - around 340 and $770 \mathrm{Ah}$ per kilogram and per liter, respectively, compared to $3800 \mathrm{Ah} / \mathrm{kg}$ for pure lithium. Thus, much effort has been expended in searches for new lithium-containing anode materials, particularly alloylike compounds with tin and silicon, both of which form lithium-rich materials (i.e., $\mathrm{Li}_{4.4} \mathrm{Sn}$ and $\mathrm{Li}_{4} \mathrm{Si}$ ). However, the very large volume changes have, just as in the case of aluminum, severely limited their extended deep cycling, which limits their practical application. Specifically, batteries, in use, can be either deep-cycled or shallow-cycled and are designed specifically for one or the other. A deep-cycled battery is one that is typically discharged to more than $50 \%$ of its capacity before being recharged - applications are golf-carts, computers, and cameras. Shallow-cycled batteries are discharged to only $10-20 \%$ of their capacity - applications include the common automotive SLI lead-acid battery and the NiMH battery in Prius HEVs. However, in a recent breakthrough, Sony showed that amorphous nanostructured tin anodes (NP-FP71 camcorder battery) can be readily recharged and have a $30 \%$ higher volumetric energy density than carbon anodes. This tin anode material has been shown to be an almost-equimolar alloy with cobalt and to contain some other critical elements such as titanium, nanosized particles of which are embedded in carbon. ${ }^{11}$ This amorphous nanosized material, shown in Figure 3, is not the answer to the anode materials challenge for large markets because of the cost and scarcity of cobalt, but it provides clues as to a possible materials approach. The challenge for the materials scientist is to fully understand why these nanosized materials work well and how to substitute an abundant lowcost material in place of the cobalt. Of even potentially greater interest would be a similar material based on silicon, with its greater energy storage capability.

A key materials challenge to making any anode work is the formation and retention of what is called the solid electrolyte interphase (SEI) layer. This film, which is formed on the sur-

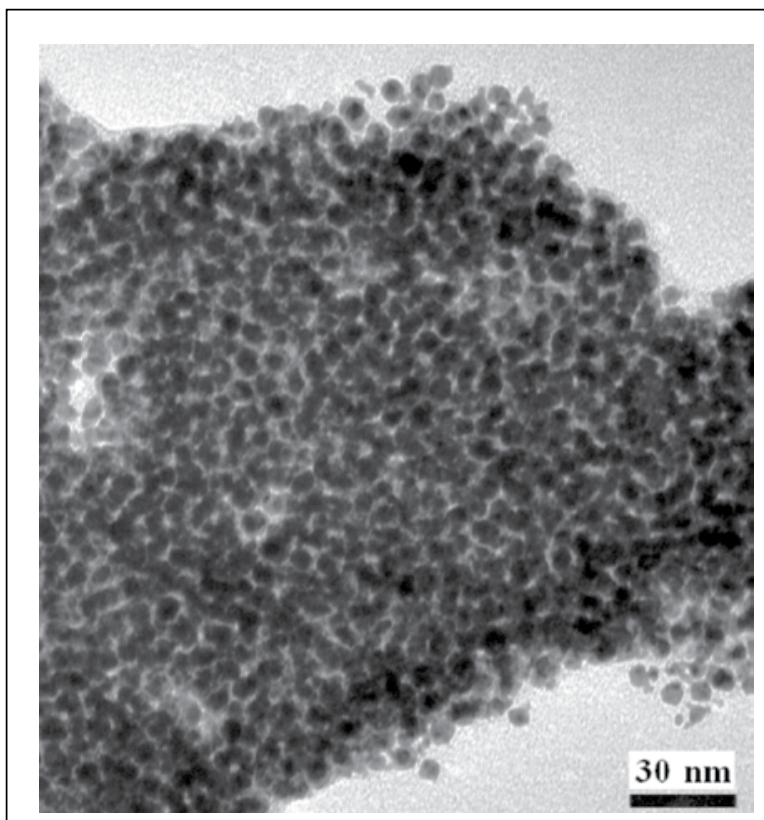

Figure 3. Transmission electron micrograph of the nanostructured amorphous tin-cobalt anode, showing nanoparticles of $\sim 6-\mathrm{nm}$ diameter embedded in a carbon matrix. From Reference 8. 
face of the anode during the first electrochemical cycle, stops the reaction between the electrolyte solvent and lithium and is critical to the safe and efficient operation of any lithium battery. It is believed to be a mix of organic and inorganic components but is presently ill-characterized - another challenge for the materials scientist. This film is one of the failure mechanisms of anode materials that expand and contract on reaction with lithium because the film will be broken and have to be reformed. The re-forming step consumes more electrolyte and creates resistive films between the anode material particles.

\section{The Cathode}

The area of potentially greatest opportunity for the materials researcher is the cathode. Here, lithium batteries are described as an example. Several materials have been used, starting with titanium disulfide TiS (commercialized in 1977), followed by lithium cobalt oxide $\mathrm{LiCoO}_{2}$ (1991) and variants with nickel and manganese $\left[\mathrm{Li}(\mathrm{NiMnCo}) \mathrm{O}_{2}\right]$, lithium manganese spinel $\left(\mathrm{LiMn}_{2} \mathrm{O}_{4}\right)$, and most recently $\mathrm{LiFePO}_{4}(2006)$. Each has its advantage and drawbacks, but none to date has much exceeded the $150 \mathrm{Ah} / \mathrm{kg}$ theoretical capacity, ${ }^{3}$ leading to practical capacities of no more than $75 \mathrm{Ah} / \mathrm{kg}$. Major materials breakthroughs are needed to significantly increase the energy storage capability while at the same time increasing lifetime and safety. A number of approaches are being considered to do this.

First, let us define the requirements of any cathode material for a lithium battery:

- the material must contain a readily reducible/oxidizable ion, for example a transition metal;

- the material must react with lithium reversibly and without major structural change to give a rechargeable cell;

- the material must react with lithium with a high free energy of reaction for high voltage;

- the material should react with lithium very rapidly on both lithium insertion and removal to give high power;

- the material should ideally be a good electronic conductor to alleviate the need for a conductive additive; and

- the material must be low cost and environmentally benign, and its elements must be plentiful in nature.

The individual redox-active material preferably provides many of these criteria, but several are opposite in character. For example, the high-voltage cathodes generally have low electronic conductivities, so that a conductive diluent must be admixed; carbon black is most commonly used, but it can lead to side reactions, particularly at high potentials, and is almost certainly not usable with the present electrolytes at $5 \mathrm{~V}$. The battery sidebar describes the complexity of the electrode. The electrochemical reactions occur predominantly at the point where electrolyte, cathode-active material, and electronic conductor are in contact. Thus, there would be many advantages in finding a material that does not require any conductive additive, such as metallically conducting $\mathrm{TiS}_{2},{ }^{4,12}$ but that operates at $3.5-4.5 \mathrm{~V}$

All commercialized rechargeable cathode materials to date basically belong to two classes: close-packed structures such as those of layered $\mathrm{TiS}_{2}, \mathrm{LiCoO}_{2}$, and cubic $\mathrm{LiMn}_{2} \mathrm{O}_{4}$ and the tunnel structures of $\mathrm{LiFePO}_{4}$. None of these have demonstrated reversible storage capacities much exceeding $500 \mathrm{Wh} / \mathrm{kg}$ under normal operating conditions.

One way to increase the storage capacity in lithium batteries is to get more than one electron per transition metal redox center. Several materials, including $\mathrm{VSe}_{2},{ }^{13} \mathrm{VOPO}_{4}{ }^{14}$ and $\mathrm{Li}_{x} \mathrm{Ni}_{0.5} \mathrm{Mn}_{0.5} \mathrm{O}_{2},{ }^{15}$ can achieve close to two electrons, as shown in Figure 4. However, in each of these cases, there is a large difference between the potential of the first reduction and that of the second reduction, which will prevent both steps from

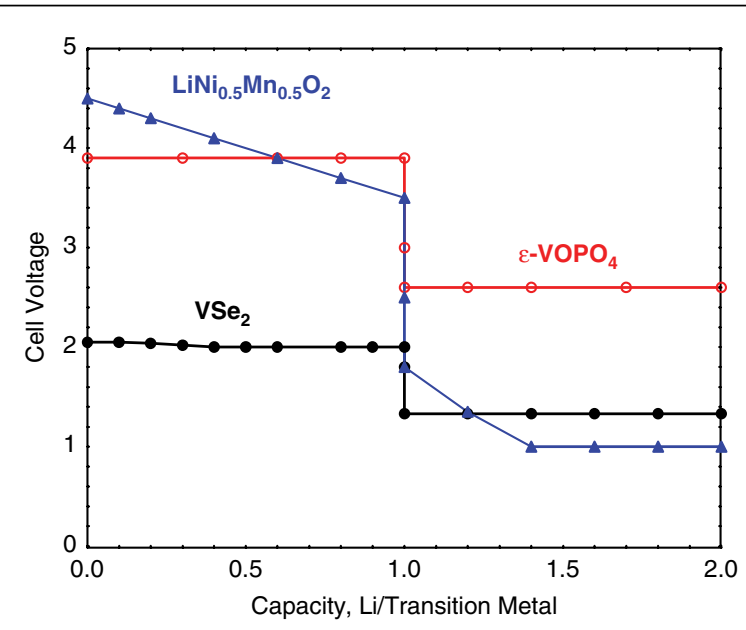

Figure 4. Examples of materials that show two-electron reduction per redox center: $\mathrm{VSe}_{2}, \mathrm{VOPO}_{4}$, and $\mathrm{LiMn}_{0.5} \mathrm{Ni}_{0.5} \mathrm{O}_{2}$.

being used in a practical battery. The second step does, however, provide protection against overdischarge. An alternative to inserting two lithium or sodium ions is to use a higher valence cation such as magnesium or aluminum, which would also considerably reduce the weight and volume of the anode. However, the ionic conductivities of these higher valence cations tend to be much reduced in solids.

A second way to increase the storage capacity is to go to cathodes with higher potentials, such as $\mathrm{LiMn}_{3 / 2} \mathrm{Ni}_{1 / 2} \mathrm{O}_{4}$, that operate at close to $5 \mathrm{~V}$. Figure 5 compares the voltage of this system with that of another close-packed oxygen lattice having equimolar amounts of nickel and manganese. ${ }^{3}$ However, there are major stability issues with the electrolyte. Today's systems are not stable much beyond $4.2 \mathrm{~V}$, with decomposition reactions occurring at higher voltages. In addition, the conductive carbon additives can react with the electrolyte salt at these potentials, forming intercalation compounds. The discovery of a high-voltage electrolyte would open a number of opportunities for increased energy storage.

A question that continually arises with the high-potential electrodes is their stability over long periods of time, for example, the $>10$ years that automotive companies demand for elec-

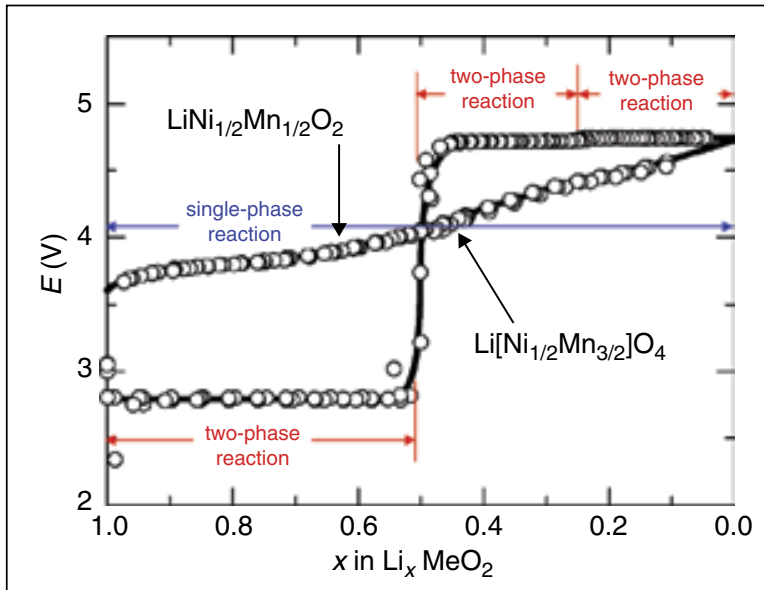

Figure 5. Lithium cell potentials of the two lithium nickel manganese dioxides with layered and spinel structures. From Reference 3. 
tric vehicles. Most of the layered oxides have potentials exceeding $4 \mathrm{~V}$ when fully charged, which means that they have an inherent high oxygen partial pressure. This oxygen is a ready combustion agent for the oxidizable components of the cell and can result in fiery explosions. $\mathrm{LiNiO}_{2}$ is the worst compound in this regard, and so nickel is always mixed with some other metal, for example, as in $\mathrm{LiNi}_{0.95-y} \mathrm{Co}_{y} \mathrm{Al}_{0.05} \mathrm{O}_{2}$. The presence of the redox-inactive aluminum prevents the complete charging of all of the nickel to the $4+$ state.

Thus, there are many proponents of using cathode materials that have lower potentials and contain firmly bound oxygen. $\mathrm{LiFePO}$, with the olivine structure, is one such material. ${ }^{16}$ The potential of this material is around $3.5 \mathrm{~V}$ versus lithium, well within the stability limits of many electrolytes; moreover, the phosphate group is extremely stable, not susceptible to being broken up on overcharge or discharge. Only under extreme discharge is the olivine structure destroyed, and even then, the phosphate group is maintained as lithium phosphate. This material, although an electronic insulator, cycles exceptionally well even at high rates when a conductive film is coated on the particles by a method involving grinding with carbon and in situ formation from carbonaceous compounds. The outstanding characteristics of this material have led to its commercialization in some high-power applications, such as professional power tools. ${ }^{17}$ Extensive studies are now underway to commercialize this technology for electric vehicles. Its energy storage is somewhat low, $590 \mathrm{Wh} / \mathrm{kg}$ theoretical at $3.5 \mathrm{~V}$. The energy storage capacity can be improved by switching to $\mathrm{LiMnPO}_{4}$ with its $4-\mathrm{V}$ potential; although this compound has been notoriously difficult to cycle, some recent work is showing high capacities comparable to that of $\mathrm{LiFePO}_{4}{ }^{18}$ Another approach to raising the energy stored is to synthesize compounds with a higher ratio of redox ion to phosphorus, thus reducing the amount of deadweight phosphate. Compounds such as lipscombite, $\mathrm{Fe}_{13} \mathrm{PO}_{4} \mathrm{O}(\mathrm{H}, \mathrm{Li})$, show some promise, ${ }^{19}$ but because they have $\mathrm{Fe}-\mathrm{O}-\mathrm{Fe}$ bonds, the discharge potential falls to around $3 \mathrm{~V}$ as a result of a lowering of the inductive effect found in the olivine structure. One way around this difficulty might be to use manganese in place of the iron, which might again give a $3.5-\mathrm{V}$ cell but with increased capacity. There are many opportunities for crystal engineering in this class of material on both the cation and the anion sites.

\section{New Battery Systems}

Lithium-ion cells have received the most attention over the past three decades, but other systems need to be researched if transformational changes are to be made. As noted earlier, sodium-sulfur cells operating at around $300^{\circ} \mathrm{C}$ have now gone commercial. The volumetric capacity of lithium cells could be significantly improved if pure lithium could be used as the anode or if a pure chalcogen (oxygen, sulfur, selenium, or tellurium) could be used as the cathode. Primary zinc-air cells have been used to power hearing aids, and if a lithium-air cell could be made operable, the energy density could be doubled over that of present-day lithium-ion cells. However, such cells have the problems of both batteries and fuel cells: the need for nondendritic replating of lithium, a reliable efficient electrocatalyst for the cathode, and a nonflooding electrolyte (i.e., an electrolyte that allows the air or other gas to get to the electrode surface where the catalyst resides). Preliminary data obtained on a lithium-air cell forming $\mathrm{Li}_{2} \mathrm{O}_{2}$ indicated reversibility, ${ }^{20}$ but there is an unacceptably high difference between the charging and discharging voltages, $4.2 \mathrm{~V}$ versus $2.6 \mathrm{~V}$, respectively, which leads to a very low efficiency. The theoretical energy density of the $\mathrm{Li} / \mathrm{Li}_{2} \mathrm{O}_{2}$ couple is around 3 kilowatt-hours per kilogram $(\mathrm{kWh} /$ $\mathrm{kg}$ ) without considering the weight of the catalyst or porous car- bon oxygen electrode-more than double that of the $\mathrm{Li}$ / $\mathrm{Li}(\mathrm{NiMnCo}) \mathrm{O}_{2}$ couple. (Note that the theoretical energy or power density typically includes only the content of the electrochemically active electrode components, for example, just the weight or volume of the $\mathrm{LiFePO}_{4}$ itself in a $\mathrm{LiFePO}_{4}$ battery. In a typical battery, the active components do not exceed $50 \%$ of the total battery weight.) However, this advantage is maintained only if a pure lithium anode is used; when carbon, $\mathrm{C}_{6} \mathrm{Li}$, is used, the theoretical energy densities are comparable, at just over $600 \mathrm{Wh} / \mathrm{kg}$. This is thus a major challenge for the electrodeposition expert. In addition to lithium and zinc, a number of other metal-oxygen couples might be viable, including aluminum and magnesium, but researchers have not devised a feasible scientific approach to date for a rechargeable battery.

Moving away from lithium altogether, systems based on sodium might be considered, but the low melting point of sodium leads to safety concerns for consumer applications. The sodium-sulfur couple has been commercialized for use with sodium- $\beta$-alumina as the electrolyte at around $300^{\circ} \mathrm{C}$, and there are possibilities of reducing the temperature of operation if suitable sulfur solvents and electrolytes can be found. In the 1970s, a number of flow redox batteries were considered, such as zincbromine by Exxon. In these batteries, which resemble fuel cells in many respects, the cathode and anode reactants are held in large tanks so that the energy storage capability can be high. Vanadium redox batteries are in commercial use now, as discussed earlier. The use of stacked bipolar plates allows for the battery pack to provide high power in a small volume; such configurations have not been considered in lithium cells because of the concern of cell imbalance leading to safety issues, but they probably deserve more attention.

\section{Electrochemical Capacitors}

Traditionally, capacitors differ from batteries by storing energy as charge on the surface of the electrodes, rather than by chemical reaction of the bulk material. As a result, the electrode does not have to undergo structural change, and capacitors have much longer lifetimes, essentially unlimited under perfect conditions. They thus also tend to have much higher rate capabilities than batteries, being almost instantaneously charged or discharged. Consequently, they are suitable for repetitive fast applications, such as regenerative braking and subsequent acceleration. However, because capacitors use only the surface of the material for charge storage, as shown in Figure 6, they are very limited in energy storage capability, with values typically from $0.1 \mathrm{Wh} /$ $\mathrm{kg}$ to $1 \mathrm{Wh} / \mathrm{kg}$ compared to well over $100 \mathrm{Wh} / \mathrm{kg}$ for lithium batteries. In addition, they provide a rather low quality of energy; that is, rather than providing energy at a relatively constant potential, they provide energy in a continuous manner from the charging voltage to $0 \mathrm{~V}$.

Capacitors are of various types. Those used in power and consumer electronic circuits are of the dielectric and electrolytic type and are mostly solid-state. They have extremely fast response times and essentially unlimited lifetimes, but store very little energy, typically less than $0.1 \mathrm{Wh} / \mathrm{kg}$. They thus have no place in applications where significant amounts of energy need to be stored. The second class of capacitors are electrochemical capacitors (ECs), ${ }^{8}$ often using high-surface-area carbon for the electrodes and sulfuric acid or acetonitrile as the electrolyte. ECs can be divided into two groups: supercapacitors and pseudocapacitors. The first of these are the electric double-layer capacitors, also known as ultracapacitors; in these, the energy is stored at the surface of the material in the double layer. Supercapacitors have improved energy storage compared to solid-state capacitors, but they still provide less than $10 \mathrm{Wh} / \mathrm{kg}$, and the cell voltages are limited to prevent the decomposition of the liquid 


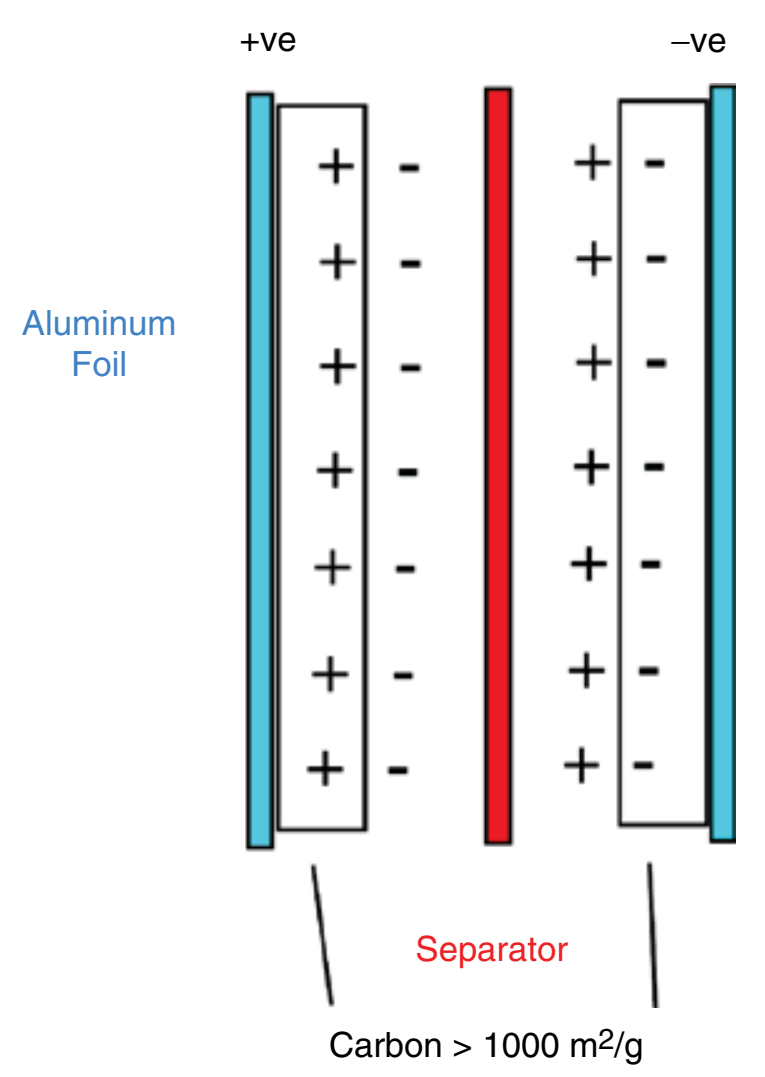

Figure 6. Schematic of energy storage in an electrochemical capacitor using high-surface-area carbon. Typical power density is $2.3 \mathrm{~kW} / \mathrm{kg}$, and typical energy density is $1.7 \mathrm{Wh} / \mathrm{kg}$.

electrolytes: less than $1 \mathrm{~V}$ for aqueous and around $3 \mathrm{~V}$ for nonaqueous-based electrolytes. The ideal applications for ECs are those demanding energy for short periods, $10^{-2}-10^{2} \mathrm{~s}$, and for thousands of cycles reliably and at low cost.

The second class of ECs comprises the pseudocapacitors, which are a hybrid between double-layer capacitors and batteries, with both the bulk and the surface of the material playing key roles. They thus can store much more energy than surface capacitors, $>10 \mathrm{Wh} / \mathrm{kg}$, but face many of the same reliability and scientific challenges as advanced batteries. Many of the same materials as used in batteries find application in pseudocapacitors, in particular, transition metal oxides such as vanadium and manganese oxides. In fact, upon reaction with lithium some crystalline compounds with flat discharge plateaus, such as vanadium pentoxide, convert to amorphous materials, as the lithium and vanadium ions become randomized in the lattice, and show sloping discharge profiles typical of a capacitor. A compound that has generated much scientific interest is hydrated ruthenium oxide, which can store around $200 \mathrm{Wh} / \mathrm{kg}$ $(750 \mathrm{~F} / \mathrm{g})$, the highest level of any capacitor. A fundamental understanding of this presently unique material is essential in order to determine its key physical and chemical behavior. This understanding might lead to a commercially viable pseudocapacitor material not containing any noble metal.

A goal for the materials scientist must be to "design capacitive storage materials" in such a way that each component preferably performs more than one function. Such a design would incorporate both chemical and surface charge storage, determine the optimum mix of each, and determine the optimum pore size distribution required to maximize the capability of the electrolyte to provide or remove the ions at a very high rate. It would also incorporate how the solvation of the charging ion changes with pore size from macropores to the extreme where an intercalation reaction occurs between neighboring sheets of the material where only a minimal or zero solvation of the ion occurs. Such a design approach would also help determine the optimum electrolyte and salt combination for high conductivity and charge transfer and address the ever-present cost issue. It is expected that new computational tools will play a key role in allowing the theory, modeling, and simulation of the complex structures, including the interfaces, demanded for the next generation of capacitors. A key challenge for any electrical energy storage device is to obtain a continuous electronic conductive pathway in the electrode so that the electrons can be inserted or removed extremely rapidly. This is even more important for pseudocapacitors, which are designed to be high-power devices. A natural extension of the porous materials researched today is to go to nanosized materials, with their inherently extreme surface areas and potentially enhanced chemical redox behavior.

Vanadium oxides are a class of materials that have been used in batteries (e.g., silver vanadium oxide in pacemakers) and because of their broad range of morphologies, these materials are beginning to garner much attention. Crystalline vanadium pentoxide, upon reaction with lithium, rapidly becomes disordered and exhibits typical capacitive-like discharge curves. Wang et al. ${ }^{21}$ have explored the morphology of nanovanadium oxides, and as shown in the Ragone plot in Figure 7, the electrochemical characteristics are strongly dependent on the morphology. (Ragone plots, in which the energy density-in $\mathrm{Wh} / \mathrm{kg}$ - is plotted against the power density, in W/kg, are typically used to compare the performance characteristics of various energy storing devices.) As shown earlier in Figure 2, the higher the energy density, the poorer the power density. These materials show behavior between those of a battery and a capacitor, and it is not unlikely that a number of future applications will use such hybrid devices.

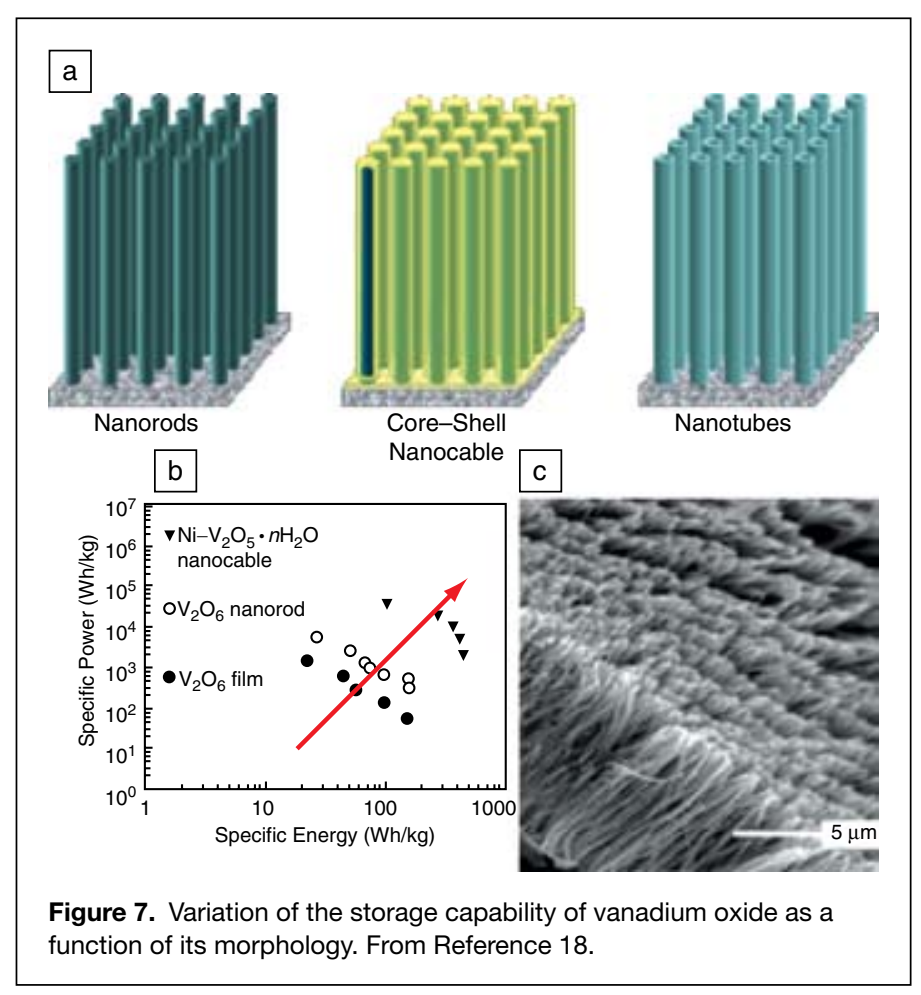




\section{Key Scientific Challenges and Opportunities Facing Batteries and Capacitors}

A number of scientific challenges and opportunities face both batteries and capacitors, whether configured for high power/low energy or lower power/high energy. Although the examples to be discussed emphasize batteries, because of the much greater research activity, they are equally applicable to electrochemical capacitors and in particular pseudocapacitors.

\section{The Role of Nanomaterials for Energy Storage}

The recent discovery of nanostructured materials, such as the tin-cobalt anode discussed earlier, has presented new opportunities for tailoring materials for energy storage. At the nanoscale, there is likely little difference, conceptually, between pseudocapacitive and storage reactions. The ability to control the structure of materials at the nanoscale adds size as a functional variable, in addition to composition and structure. These materials exhibit new phenomena beyond that associated with just the larger surface areas, such as the amelioration of the impact of volume expansion in the tin upon reaction with lithium and the apparent change of the phase diagram of $\mathrm{LiFePO}_{4}$. However, the lack of a fundamental understanding of how thermodynamic properties, such as phase coexistence, change at the nanoscale is in direct contrast to the wealth of information available on the physical properties of nanomaterials, such as magnetic, optical, and electronic properties.

Many fundamental questions remain to be answered about how materials at the nanoscale significantly differ from those in the bulk. How can the different properties of nanoparticles and their composites be used to increase the power and energy efficiency of battery systems? How does the chemical reactivity of materials differ at the nanolevel, and how do the ionic and electronic conductivities couple and behave at the nanolevel? How do we control the morphology and particle size of nanomaterials to optimize transport behavior as well as packing density? An issue with all nanocomposites is their packing density, which might severely limit the volumetric storage capacity.

A challenge to be solved is the development of low-cost, high-volume synthesis and fabrication techniques for nanocomposites, which must have improved safety characteristics for their use in practical battery and pseudocapacitor systems. Recently, a number of groups have shown the ability to produce nanosized $\mathrm{LiFePO}_{4}$ by solvothermal, high-temperature firing or molten-salt methods. Figure 8 shows the excellent power capa-

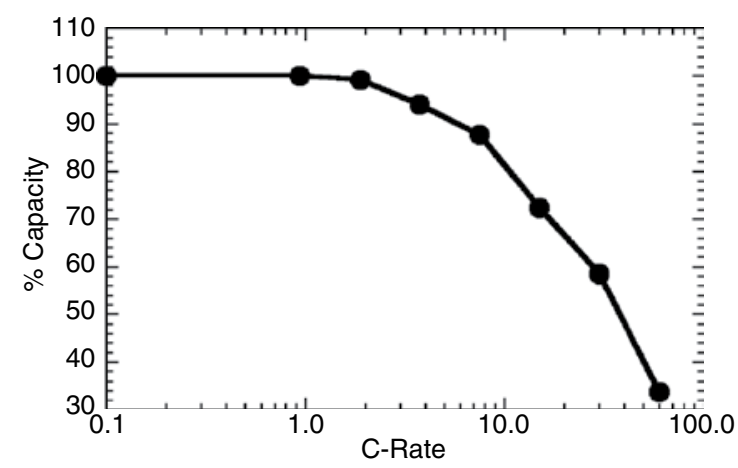

Figure 8. High capacity of a $\mathrm{LiFePO}_{4}$ cathode is maintained even at high rates when it is nanosize. The term $\mathrm{C}$-rate refers to the time it takes to discharge the cell: $1 \mathrm{C}$ takes $1 \mathrm{~h} ; 2 \mathrm{C}$, $30 \mathrm{~min}$. Data from Reference 19. bility of $\mathrm{LiFePO}_{4}$ made by a solvothermal approach, when mixed with carbon black. ${ }^{22}$ The company HPL has similarly shown much improved electrochemical behavior of solvothermal $\mathrm{LiMnPO}_{4}$ when ground to nanosize with carbon. ${ }^{18}$

A key recent finding that carbon nanotubes, with their extremely high aspect ratio, reach the conductivity percolation threshhold in mixtures at levels of only $0.1 \mathrm{wt} \%$ could dramatically reduce the weight and volume of the conductive diluent that needs be added to cathode mixtures. ${ }^{23}$ This could explain the improved electrochemical behavior report for $\mathrm{LiFePO}_{4}$ formed hydrothermally in the presence of carbon nanotubes. ${ }^{24}$

\section{Multipurpose Materials in Energy Storage}

As described in the battery sidebar, batteries and electrochemical capacitors contain many components that are critical to performance but that do not contribute to the energy or power density and instead reduce both the gravimetric and volumetric capacities. Such components include the conductive additive and binders that are used in both electrodes, as well as the critically important electronically insulating separator. A challenge to the materials scientist is to design materials that are multipurpose, for example, a conductive binder or a separator that can also serve as a cell cutoff in the event of thermal runaway. Titanium disulfide is a conductive diluent that could also be used for overdischarge protection. Another area for exploration is the use of current collectors that could be used in a bipolar cell configuration, where one side is the anode and the other the cathode. In such batteries/capacitors, the cells are simply stacked to deliver the desired battery voltage with no external connections between the individual cells.

\section{The Role of Theory and Modeling in Electrical Energy Storage}

The rational design of novel electrical energy storage systems with high energy and power density will benefit from a combined experimental and theoretical approach. The latter requires the calculation of all the properties of electroactive materials and the microstructures in which they are embedded. A revolutionary advance, for example, would be the design and/ or prediction of materials that can react with multiple electrons per redox couple within, say, a 1-V window. Although batteries and electrochemical capacitors operate on different time scales, with the former using slower reactions involving the bulk of the material and the latter faster interfacial reactions, many of the issues are common, particularly for pseudocapacitors, including the behavior of ions and solvents confined in pores of various dimensions.

Significant progress has already been made since the pioneering work of the Ceder group ${ }^{25}$ that has allowed the calculation of the redox potentials and ion mobilities of several important cathode materials. Mesoscale modeling has allowed the simulation of transport and mechanics in complex architectures of packed agglomerates, ${ }^{26}$ but a full predictive theory of chemical energy storage systems still requires fundamental research and methodological developments in several key areas. Progress in these key areas will eventually allow higher energy and power at lower cost and will dramatically accelerate innovation in new materials.

- Modeling the interfaces. The solid electrolyte interphase (SEI) layer formed between the electrodes and the electrolyte is critical to the performance, life, and safety of electrochemical devices. This complex, continually changing interface is very poorly understood and not well described in mathematical performance models. It will be absolutely essential to fully understand this living layer in order to design the long-lived cells demanded by electric vehicles. Such an 
undertaking will demand a joint effort between the theorist and novel in situ characterization tools.

- Modeling ion and electron motion. The prediction of the motion of ions and electrons in crystalline and amorphous lattices, as well as in constrained liquids, is particularly important for chemical energy storage. These predictions then need to be combined to give a picture of the whole system, which will require the models to be significantly complex to capture ion and electron transport in multicomponent electrolytes and complex multiphase microstructures. The high anisotropy of many electrode materials such as the one-dimensional ion-conducting olivines must be considered. This anisotropy is often associated with extreme electronic insulator characteristics, because strong bonding groups such as phosphates are likely to lead to safe electrodes. The olivine $\mathrm{LiFePO}_{4}$ is one such example where the strong coupling between the localized polarons and the mobile lithium ions had to be taken into account. ${ }^{27}$

- Modeling kinetics and mechanism of phase changes. The mechanism by which new phases are nucleated and translate through electrode materials is poorly understood. How the phase change occurs in the $\mathrm{LiFePO}_{4} / \mathrm{FePO}_{4}$ system and what limits its translation through the crystallites is not clear. What is the impact of crystallite morphology - does a thin platelet show maximum phase transition rates, or do defects catalyze the nucleation and motion of phase fronts?

- Modeling nanoscale materials and surfaces. Modeling is needed to determine the length scale at which the kinetics and thermodynamics of nanoparticles become significantly different from those of the bulk and the surface and to predict how the properties might differ particularly in the length scale from $10 \mathrm{~nm}$ to $150 \mathrm{~nm}$. Such information would help guide the experimentalist in targeting the most important materials. Today, even the relative thermodynamic stabilities of the different known bulk phases of $\mathrm{FePO}_{4}$ are not known. The challenge to the theorist is to predict the chemical structure of the next generation of phosphates that have capacities approaching $300 \mathrm{Ah} / \mathrm{kg}$ to allow batteries with a theoretical storage capability of $1 \mathrm{kWh} / \mathrm{kg}$.

- Design of electrode structures. As noted in the battery sidebar, control of the microstructure of the complex composites making up electrodes is critical to the long-term operation of the battery. Modeling is needed to prescribe the optimum particle size and morphology, the optimum mixture of these particulates with the conductive diluent and binder, the optimum porosity, and the impact of electrodes swelling and contracting on reaction. A full understanding of these parameters will allow optimum design for energy storage and power output.

\section{The Characterization of Materials and Cells in Electrical Energy Storage}

Batteries and pseudocapacitors are living systems, with ions in the electrodes other than the mobile ion, such as lithium, moving as a function of discharge and charge state. These motions often cause phase changes to occur, and it is important to understand the mechanism so that the materials can be optimized for all conditions of charge. For example, in bulk micron-size $\mathrm{LiFePO}_{4}$, the two phases $\mathrm{FePO}_{4}$ and $\mathrm{LiFePO}_{4}$ are in equilibrium, and conversion between them is believed to occur by a reaction front moving along the ac plane with an amorphous disordered nanophase at the interface, as determined by a transmission electron microscopy (TEM) study ${ }^{28}$ on a hydrothermally grown single crystal. In contrast, $\mathrm{Li}_{x} \mathrm{FePO}_{4}$ under $20 \mathrm{~nm}$ is thought to possibly react by a simple singlephase diffusion mechanism. However, the mechanism for the mixed $\mathrm{LiFe}_{1-y} \mathrm{Mn}_{y} \mathrm{PO}_{4}$ material is still not understood, as it is a multiple two-phase system.

An even more complex class of cathode material is the layered oxide, $\mathrm{Li}_{1+y}\left[\mathrm{Ni}_{z} \mathrm{Mn}_{z} \mathrm{Co}_{1-2 z}\right]_{1-y} \mathrm{O}_{2}$. In this layered structure, alternating layers are ideally occupied by the transition metal and lithium, respectively, when $y=0$. However, for essentially all compositions, nickel migrates to the lithium layer, and a comparable amount of lithium is found in the transition metal layer. As $y$ increases, the lithium content in the metal layer increases. Recent data suggest that the nickel ions migrate, and possibly the other transition metals do as well, as the material is charged in a cell. In addition, when the material is completely charged, the stacking of the $\mathrm{MO}_{2}$ sheets can rearrange to give hexagonal close-packed sheets. This disruption of the stacking sequence from $\mathrm{ABCA}$ to $\mathrm{ABAB}$ results in a marked loss of capacity; this is the reason that $\mathrm{LiCoO}_{2}$ is not charged for values of $x$ much less than 0.5. A little residual nickel in the lithium layer might pin the layers and prevent this change in stacking sequence. It is therefore critical to be able to thoroughly characterize the electrodes, preferably in situ, which will demand much-enhanced characterization capabilities with simultaneous measurement techniques. For example, a combined x-ray and neutron diffraction analysis shows that nickel migrates to the lithium layer, and nuclear magnetic resonance spectroscopy can determine the presence of lithium in the transition metal layer, the identities of its neighbors, and also whether it is in octahedral or tetrahedral sites. ${ }^{29,30}$ Magnetic susceptibility measurements have shown the presence of nickel ions in the lithium layer and how these interactions change as the lithium content is lowered on charging. ${ }^{31}$

Even more challenging will be the characterization of advanced nanomaterials, which are often amorphous or at best poorly crystalline. More advanced tools will be required, such as pair distribution function (pdf) analysis of structural data as exemplified by its application to the Sony Sn-Co anode ${ }^{11}$ and on vanadium oxide nanoscrolls. ${ }^{32}$

In all studies of electrochemical cells, in situ measurements will allow for an understanding of dynamic phenomena and processes at the electrode-electrolyte interface as well as within the cathode and anode materials during charge-discharge cycles. New tools will need to be devised to permit the functional state of the cell to be determined during operation. Furthermore, new computational tools will be required to assist in the interpretation of advanced structural and spectroscopic measurements, such as in the generation of possible structures of amorphous materials and nanomaterials.

\section{The Electrolyte}

A major cost factor and limitation for the next generation of batteries and capacitors is the electrolyte. At this time, there is a major lack of understanding of the molecular interactions occurring within the electrolyte and at the solid interfaces with which the electrolyte is in contact. Moreover, these interfaces can be on the external surface of the electrodes or in the complex internal porous structure. An advanced electrolyte, whether for a battery or for an electrochemical capacitor, will need the following characteristics, compared to today's systems:

- higher ionic conductivity to give high cycling rates over a wide range of temperature, ideally from $-30^{\circ} \mathrm{C}$ to $100^{\circ} \mathrm{C}$;

- higher stability, both chemical and electrochemical, to allow for higher voltage systems and to increase safety;

- higher compatibility with other cell components, both for better wettability and for lower corrosion/reaction rates; and

- lower cost and decreased environmental impact.

Today, lithium batteries use electrolytes containing an $\mathrm{LiPF}_{6}$ salt dissolved in a mixed carbonate solvent, whereas the highvoltage capacitors use quarternary ammonium salts dissolved 
in acetonitrile or an organic carbonate. There is a desire to move away from the $\mathrm{LiPF}_{6}$ salt, which can produce hydrogen fluoride (HF) in even traces of moisture. This HF can cause dissolution of the cathode metals, the atoms of which then migrate to and react with the lithium-graphite anode, causing significant loss of capacity. Boron-based salts are of interest because of their higher stability, but in some cases, the SEI layers they form are too resistive. LiBOB and its fluorinated analogs are of particular interest and might lead to completely new systems over the next decade. Another research opportunity lies in ionic liquids. These are salts that are liquid under ambient conditions and do not need any solvent for operation. They also tend to have low vapor pressures and to be nonflammable, but they might be too reactive to be used with lithium and some cathode materials with which they can form complexes. However, the present materials might find application in high-power batteries, such as the dual spinel $\mathrm{Li}_{4} \mathrm{Ti}_{5} \mathrm{O}_{12} / \mathrm{LiM}_{2} \mathrm{O}_{4}$ system, or in electrochemical capacitors. This is just the beginning of new opportunities for the electrolyte chemist, and major breakthroughs can be anticipated. For further information, Reference 3 provides excellent reviews on electrolytes and separators.

\section{Summary}

Electrical energy storage is crucial for the effective proliferation of an electric economy and for the implementation of many renewable energy technologies. Transformational changes in both battery and capacitor science and technology will be required to allow higher and faster energy storage at the lower cost and longer lifetime necessary for major market enlargement. Most of these changes require new materials with larger redox capacities that react more rapidly and reversibly with cations such as lithium.

\section{Acknowledgments}

The work at Binghamton was supported by the U.S. Department of Energy, through the BATT program at Lawrence Berkeley National Laboratory. This article also relies on the U.S. DOE workshop presentations held in April 2007, on its subsequent report, and on discussions held with many colleagues. In particular, I thank Bruce Dunn for helpful discussions, Imre Gyuk of U.S. DOE for much useful information on applications, and T. Ohzuku for providing Figure 5.

\section{References}

1. K.H. LaCommare, J.H. Eto, Understanding the Cost of Power Interruptions to U.S. Electricity Consumers (Energy Analysis Department,
Lawrence Berkeley National Laboratory, University of California-Berkeley, Berkeley, CA, 2004; http://certs.lbl.gov/pdf/55718.pdf) (accessed January 2008).

2. Dinorwig Power Station, http://www.fhc.co.uk/dinorwig.htm (accessed January 2008).

3. M.S. Whittingham, R.F. Savinell, T. Zawodzinski, Eds., "Batteries and Fuel Cells", in Chem. Rev. 104, 4243 (2004).

4. M.S. Whittingham, Prog. Solid State Chem. 12, 41 (1978).

5. D. Linden, T.B. Reddy, Handbook of Batteries (McGraw Hill, New York, ed. 3, 2001).

6. J. M. Tarascon, M. Armand, Nature 414, 359 (2001).

7. Basic Research Needs for Electrical Energy Storage (Office of Basic Energy

Sciences, U.S. Department of Energy, Washington, DC, 2007).

8. R. Kötz, M. Carlen, Electrochim. Acta 45, 2483 (2000).

9. G.G. Libowitz, M.S. Whittingham, Materials Science in Energy Technology (Academic Press, New York, 1979).

10. S. Flandois, B. Simon, Carbon 37, 165 (1999).

11. Q. Fan, P.J. Chupas, M.S. Whittingham, Electrochem. Solid-State Lett. 10 (12), A274 (2007)

12. M.S. Whittingham, Science 192, 1126 (1976)

13. M.S. Whittingham, Mater. Res. Bull. 13, 959 (1978).

14. Y. Song, P.Y. Zavalij, M.S. Whittingham, J. Electrochem. Soc. 152, A721 (2005).

15. C.S. Johnson, J.S. Kim, A.J. Kropf, A.J. Kahaian, J.T. Vaughey, L.M.L. Fransson, K. Edström, M.M. Thackeray, Chem. Mater. 15, 2313 (2003).

16. A.K. Padhi, K.S. Nanjundaswamy, J.B. Goodenough, J. Electrochem. Soc. 144, 1188 (1997).

17. A123; www.a123systems.com (accessed January 2008).

18. T. Drezen, N.-H. Kwon, P. Bowenb, I. Teerlinck, M. Isono, I. Exnar, J. Power Sources 174, 949 (2007).

19. Y. Song, P.Y. Zavalij, N.A. Chernova, M.S. Whittingham, Chem. Mater. 17, 1139 (2005).

20. T. Ogasawara, A. Débart, M. Holzapfel, P. Novák, P.G. Bruce, J. Am. Chem. Soc. 128, 1390 (2006).

21. Y. Wang, K. Takahashi, K.H. Lee, G.Z. Cao, Adv. Funct. Mater. 16, 1133 (2006).

22. D.-H. Kim, J. Kim, Electrochem. Solid-State Lett. 9, A439 (2006).

23. A. Windle, private communication.

24. J. Chen, M.S. Whittingham, Electrochem. Commun. 8, 855 (2006).

25. F. Zhou, M. Cococcionic, C. Marianetti, D. Morgan, M. Chen, G. Ceder, Phys. Rev. B 70, 235121 (2004).

26. C.-W. Wang, K.A. Cook, A.M. Sastry, J. Electrochem. Soc. 150, A385 (2003).

27. T. Maxisch, F. Zhou, G. Ceder, Phys. Rev. B 73 (2006).

28. G. Chen, X. Song, T.J. Richardson, Electrochem. Solid-State Lett. 9, A295 (2006).

29. J. Breger, N. Dupre, P.J. Chupas, P.T. Lee, T. Proffen, J. Parise, C.P. Grey, J. Am. Chem. Soc. 127, 7529 (2005)

30. C.P. Grey, N. Dupre, Chem. Rev. 104, 4493 (2004).

31. N.A. Chernova, M.M. Ma, J. Xiao, M.S. Whittingham, J. Breger, C.P. Grey, Chem. Mater. 19, 4682 (2007)

32. V. Petkov, P.Y. Zavalij, S. Lutta, M.S. Whittingham, V. Parvanov, S. Shastri, Phys. Rev. B69, 085410 (2004).

\title{
Electrical Energy Storage Using Flywheels
}

\author{
M. Stanley Whittingham (Binghamton University, USA)
}

Flywheel energy storage systems use the kinetic energy stored in a rotor; they are often referred to as mechanical batteries. On charging, the flywheel is accelerated, and on power generation, it is slowed. Because the energy stored is proportional to the square of the speed, very high speeds are used, typically 20,000-100,000 revolutions per minute (rpm). To minimize energy loss due to friction, the rotors are spun in a vacuum and use magnetic bearings. The rotors today are typically made of high-strength carbon composites. One of the main limits to flywheels is the strength of the material used for the rotor: the stronger the rotor, the faster it can be spun, and the more energy it can store. However, if the strength is exceeded, 